Thorax (1948), 3, 161.

\title{
POSTURE IN THORACIC SURGERY
}

BY

\author{
A. I. PARRY BROWN
}

From the Thoracic Surgical Unit, Harefield, Middlesex

It is important in thoracic surgery to find a position upon the operating table which will combine good surgical access with the minimum interference with respiration. Present practice favours a postero-lateral approach for most thoracotomies, and for this the patient is placed with the good side of the chest under. The lateral position is maintained by a pad against the front of the chest and by pelvic and buttock supports which prevent rolling or slipping if the table is tilted. A padded wedge or pillow is sometimes placed under the thorax so as to bow the chest, opening the rib spaces on the upper side and so facilitating the exposure. Sellors' "chest-rest" combines the wedge and the anterior support in one piece. This position gives an excellent exposure for the surgeon but interferes with respiratory exchange.

It has been stated, as a result of the investigations of Björkman and Jacobeus in bronchospirometry (Björkman, 1934), that with a patient lying in the lateral position the lower lung has the greater respiratory exchange. This appears to be a fortuitous advantage accruing from a position which has been adopted because of its surgical convenience ; but when the chest is open the tidal air into the lower lung is reduced; and, unless methods are adopted to assist ventilation, anoxia develops.

\section{Pleural Pressure}

Sellors has investigated the pleural pressures of patients with established pneumothoraces lying in different postures. When such a patient is moved from the supine to the lateral position, so that the pneumothorax is uppermost, the intrapleural pressure becomes more negative, and to restore it to its former level air must be introduced into the pleural cavity. In one series of twelve cases the volume of air that had to be introduced varied from 500 to $800 \mathrm{c.cm}$. with an average of nearly $700 \mathrm{c.cm}$. in eleven of the cases. The exception was a case of old pleurisy with a rigid mediastinum, in whom $250 \mathrm{c.cm}$. restored the pressures. If the pneumothorax is on the underside the intrapleural pressure is increased and can be corrected by aspiration of air, and when a patient who has had a pneumonectomy is turned from the lateral to the supine position at the end of the operation the pleural pressure on the operation side is also increased, so that in a well-grown adult it may be necessary to aspirate 600 to $800 \mathrm{c.cm}$. of air in order to produce normal readings and to centralize the mediastinum. These conditions show an increase in the capacity of the upper hemithorax at the expense of the lower in the lateral position.

Penman (1941), in an investigation of cases who had " mediastinal flap," and who were treated by artificial pneumothorax, found the same thing, and in treating mediastinal displacement he used low pressures and nursed the patients as far as possible on the side with the pneumothorax undermost.

\section{Disadvantages of the Lateral Position}

Blair (1941) has discussed the displacement of the mediastinum which results from the action of gravity when adopting the lateral position (Fig. 1).

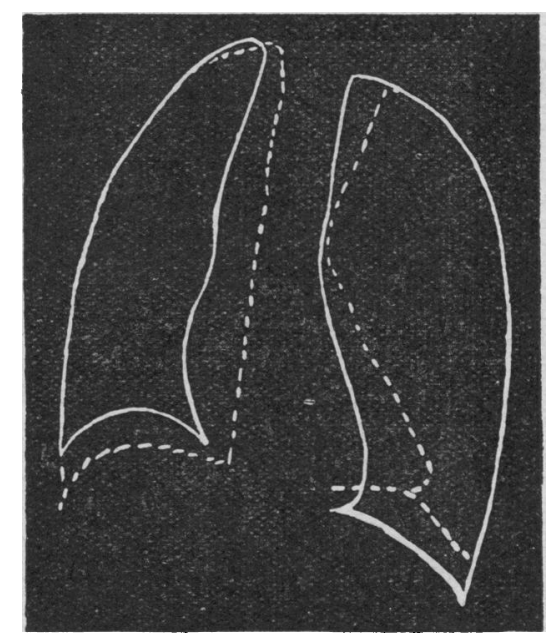

FIG. 1.-Superimposed tracings to show a mediastinal displacement with the patient supine and on the left side. The continuous line shows the outline of the mediastinum with the patient supine; the broken line shows it with the patient lying on the left side. (By kind permission of the Editor, Proceedings of the Royal Society of Medicine.) 
His observations were made on radiographs of a number of normal individuals whose posture was altered ; they were made at the end of a normal expiration, as the conditions in this phase are least affected by variations in the depth of respiration, thus permitting a fairer comparison of the supine and lateral positions. They showed that when the patient was moved from the supine to the lateral position there were changes in the position of the mediastinum and of the diaphragm on both sides. The mediastinum moved towards the lower side, and the diaphragm rose and became more arched on the under side, whilst it was flattened on the upper side.

In the lateral position the diaphragm has a greater potential excursion on the lower side, for on the upper side it is already in the inspiratory position. The mediastinum moves towards the mid-line on inspiration, thus increasing the volume of the lower hemithorax but decreasing that of the upper. This accords with the bronchospirometric finding that the under lung is better ventilated, but when the chest is opened the picture is altered. The mediastinum moves with each respiration in response to the differences in pleural pressure on the two sides. On the open or upper side the pleural pressure remains atmospheric, but on the closed side the pressure becomes lower during inspiration. The mediastinum moves into the lower hemithorax with each inspiration, and towards the mid-line during expiration. This paradoxical movement has the result that for any given excursion of the ribs and diaphragm less air than usual is drawn into the chest. A wedge placed under the chest impairs the movement of the ribs and renders the position still more unfavourable for adequate respiratory exchange. Although the anaesthetist can control this paradoxical mediastinal movement by controlled or assisted respiration, and can limit the gravitational shift of the mediastinum by positive pressure in the under lung, it makes an extra and unnecessary difficulty.

The second disadvantage of the lateral position is that pus, blood, or bronchial secretion extruded from the diseased lung gravitates into the sound under lung. There they can produce bronchial obstruction, collapse, and infection. Many procedures have been adopted to prevent this soiling of healthy lung. Spinal and local analgesia had an extensive trial. The purpose of these methods was to preserve an active cough reflex and the full ciliary movement of the bronchial epithelium, and so to enable the patient to clear his own air passages and protect the sound lung. These methods failed because of the mechanical impossibility of obtaining an effective expulsive cough in the presence of an open pneumothorax. Attempts to raise the intrabronchial pressure, which normally provides the expulsive force behind the closed glottis, are dissipated in a paradoxical distension of the exposed lung. As the cough is not effective, the stimulus which initiated it remains active and produces further bouts of coughing which are exhausting to the patient and disturbing to the surgeon.

\section{Postural Drainage}

The customary method of removing excessive secretions is by adopting such a posture that gravity assists the drainage. In most cases, particularly in bronchiectasis of the lower lobe, this requires the prone position with the head lowered so that secretions flow up the trachea into the mouth. Such postural drainage is a routine part of the pre-operative treatment, and should be carried out immediately before the patient is brought to the operating theatre. In spite of these precautions large volumes of pus may be expelled from the lung of a patient suffering from bronchiectasis during the induction of anaesthesia. The flow of pus is increased by collapse of the lung as the pleura is opened, and later by actual manipulation and squeezing of lung tissue during dissection.

It is possible to secure some protection for healthy areas of lung by lowering the head and shoulders. The protection is more effective if the left side is uppermost, owing to the greater obliquity of the left main bronchus. Nosworthy (1941) has discussed this position and has shown how steep the inclination of the table must be to protect the under lung. In calculating the angle of tilt required allowance should be made for the displacement of the mediastinum from the pull of gravity. This lowers the bifurcation of the trachea and makes the tip less effective. We find the "head-down" tilt has to be so steep as to make surgical access difficult and slipping of the patient in the course of the operation very probable.

\section{INTRABRONCHIAL SUCTION}

Intrabronchial suction by a long fine gum elastic catheter passed down the endotracheal anaesthetic tube is a valuable adjunct to the use of posture in clearing the airway, but there are practical disadvantages in its use. The smooth administration of the anaesthetic is interrupted; bouts of coughing may be initiated unless the patient is at a deeper plane of anaesthesia than is otherwise required for the operation; the procedure can cause haemorrhage, particularly in cases of carcinoma or adenoma ; it may fail where 
the secretions are so viscid that they cannot be sucked through the fine tube, or so copious as to require continuous suction. If, by over-zealous attempts to remove all the secretions, the catheter is passed into small bronchi of a diameter approximating to its own, all the air can be exhausted from a lobule, thus causing atelectasis.

\section{BLOCKING THE BRONCHUS}

Blocking the bronchus has been used to overcome the difficulties referred to above. In principle there are two possible methods. One is to administer the anaesthetic gases through an endobronchial catheter into the good lung whilst a balloon around the tube isolates the diseased lung. The alternative is to block the bronchus of the diseased lobe or lung whilst the respiratory exchange is continued through an endotracheal tube. An advantage of one-liung anaesthesia is that the mediastinum can be maintained in its normal position by controlled respiration with intermittent positive pressure without ballooning the lung in the open hemithorax. If it is necessary to maintain positive pressure during expiration as well as inspiration, to prevent the sag of the mediastinum, the change of the alveolar air will be less complete with each respiration and the pulmonary circulation may be impaired. When attempting a right endobronchial anaesthetic, even if Magill's special tube is employed, it is difficult to achieve isolation of the left lung without obstructing the right upper lobe bronchus. Unless a pneumothorax has been produced preoperatively, the pulmonary circulation will continue through the non-ventilating but still distended lung, causing anoxia, and the bulk of the lung may impair access to the hilum. Secretions are retained in the lung and may be squeezed past the balloon into the airway during the course of the operation. The retention of the secretions renders the method unsuitable for lobectomy, as it would allow contamination of the remaining lobes on the diseased side.

The alternative has its disadvantages. If isolalation is achieved by tamponage, secretions are retained and there is danger of soiling the pleura during dissection, and some accident of surgical manipulation may displace the tampon and so obstruct the airway. If it is achieved by introducing $\bullet$ fine drainage tube carrying a balloon into the bronchus of the diseased lobe or lung secretions can be removed continuously although the diseased lung is isolated from the airway; the earlier drains of this type slipped from position too easily. The gauze-covered balloon introduced by Thompson overcomes this difficulty. The distal end of the apparatus is made of metal and the balloon which surrounds this metal end can be inflated to a considerable tension because the metal prevents the occlusion of the tube and the net strengthens the balloon. When the balloon has been tightly inflated with water, the gauze prevents it from slipping in the bronchus. The introduction of the tube to its correct position in the bronchial tree presents difficulties; it is most readily done under local analgesia, as the procedure is then unhurried, but the distension of the balloon can produce coughing in patients who have borne the bronchoscopy with equanimity. If general anaesthesia is used, there is less time for the accurate positioning of the balloon and some risk of contaminating healthy lung before the diseased area has been isolated ; but by the use of curare this has been made simpler. Apart from the difficulties of introduction, and mechanical accidents such as deflation of the balloon, there are some cases for which these methods are inapplicable. The Thompson balloon can only be passed through an adult bronchoscope, and thus the technique cannot be used for children. In patients with such multiple lesions as bronchiectasis in the left lower lobe and in the lingula of the upper lobe, only partial protection can be obtained.

\section{Operating with the Diseased Side Lower}

In attempting to find a technique suitable for these cases we had to fulfil three conditions: the surgical exposure must be adequate, the mediastinum should be as little displaced as possible, and secretions should be kept away from healthy lung. Since the ordinary lateral position failed to satisfy the last two of these conditions, we were at first impelled to attempt its converse by supporting the patient in a tubular frame and operating with the diseased side lower. This has been done for the drainage of empyema, where only a small posterior exposure is required. It gives excellent protection against flooding of the bronchial tree if there is an unsuspected bronchopleural fistula. We were unable to fix the patient so as to give an adequate exposure for a major thoracotomy.

\section{The Sitting Position}

The sitting position with chest and head supported avoids gravitational displacement of the mediastinum; ' and secretions, retained in the diseased areas, do not flow into the air passages. This position serves admirably for drainage of empyemata-particularly if there is a bronchopleural fistula-and in the treatment of a lung 
abscess, but it is not well adapted to more severe operations because patients are liable to a marked fall in blood pressure.

\section{ANTERIOR APPROACH WITH THE PATIENT SUPINE}

The anterior approach with the patient supine has had a period of usefulness. There is no gravitational displacement of the mediastinum. There is no "under-lung" to fill with secretions, but the slope of the trachea is up from the bifurcation to the larynx, necessitating the use of suction to remove sputum. Exposure is, however, limited, closure of the wound is difficult, and the scar tends to be ugly.

\section{The Prone Position}

The exposure we have gradually developed and now use for all our "wet " cases is with the patient prone (Fig. 2). The plane of the mediastinum is

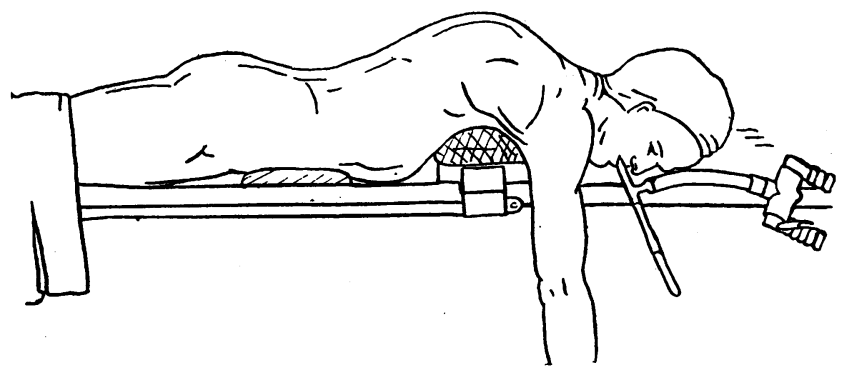

FIG. 2.-The position advocated for a right thoracotomy. of the incision hangs downwards over the edge of the table, thus drawing the scapula away from the field and facilitating the approach. If a shoulder rest is used to prevent the patient from sliding up the table, the scapula cannot swing clear of the chest and the ribs are not easily retracted. The other arm lies conveniently on the table beside the patient and may be used for intravenous injections.

When the patient has been properly placed the table should be tilted so that the lumbar and lower dorsal vertebrae are horizontal whilst the upper dorsal and cervical vertebrae are flexed and have a downward curve. Although the cervical vertebrae are flexed, the head is extended on the atlantooccipital joint and turned to the side of operation. This rotation and lateral flexion of the head straightens the line of the trachea and the bronchus to the working lung. If the head is rotated away from the open hemithorax, traction on the hilum may kink the main bronchus on the other side, causing grave respiratory obstruction. The inclination of the trachea depends mainly on the flexion of the neck. If the chest rest is not high enough to permit full flexion, then the drainage of the trachea will be inefficient.

Two rests are so placed that the abdomen is clear of the table and the respiratory movement of the diaphragm can occur without lifting the weight of the patient. The other respiratory movement which occurs is a lifting of the ribs on the closed side with each inspiration. If this is vertical, and it is not displaced by gravity. Our aim in the control of secretions has been to let them flow up the trachea into the mouth. In our early cases we made the table into a wedge, over which the patient lay prone, with flexed hips. The weight of the legs counterbalanced that of the body. This produced an effective downward inclination of the trachea from the bifurcation to the larynx, and enabled secretions to flow up to the mouth. The surgical approach in this position was complicated by the Trendelenburg slope as well as the posterior incision, and we have found that effective drainage can more easily be obtained by the use of two supports. A well-filled pillow under the pelvis holds the patient two or three inches clear of the table. The chest rest should be four inches thick and of sufficient area to take the weight of the patient ; it should be so placed that its upper edge reaches the manubrium sterni as the patient lies over it. The side for operation should slightly overhang the edge of the table, as this allows a longer incision. The arm on the side restrained by the weight of an assistant's hands, bigger pressures are necessary to ventilate the patient and ballooning of the lung on the open side may result.

The anaesthetic is conveniently given through a large cuffed oro-tracheal tube. Although in this position the wide unilateral pneumothorax affects respiration less adversely than in the lateral position, it is best to ensure adequate ventilation by using controlled or assisted respiration. The secretions which gravitate along the endotracheal tube towards the mouth can be collected by using a T-connexion between the anaesthetic apparatus and the endotracheal tube. A test-tube connected to the transverse arm of the T-piece serves as a receptacle for the sputum. Viscid secretions which are adhering to the walls of the endotracheal tube can be removed by passing a fine gum-elastic suction catheter.

There are some differences in purpose and technique between this position and that of Overholt (1946). His expressed purpose was to retain 
the secretions in the cavities of the lung until the bronchus had been divided, whereas we encourage continuous free drainage of the secretions. We have had to abandon the use of shoulder rests for the reasons given above.

The position we advocate overcomes many of the difficulties encountered in the standard lateral position, since the affected lung is continually drained by gravity during the operation and there is greater stability of the mediastinum. The objections are the more limited exposure of the chest wall as compared with that obtained by lateral thoracotomy, and the necessity for a reorientation of the surgical anatomy.

An adequate surgical exposure is obtained by starting the incision close to the spine and carrying it round to the anterior axillary line, for the hilum lies near the surface in this position; but the division of pleural adhesions, especially over the diaphragm, is more difficult than with the wide exposure of a lateral thoracotomy. The dissection of the posterior aspect of the hilum is easy and the bronchus can be isolated first. . The pulmonary veins have frequently to be ligated before the artery, which is theoretically wrong as leading to engorgement of the lung to be excised, but this seems to be unimportant practically.
We pass a bronchoscope postoperatively, and usually the bronchi on the good side are clear of secretions. The importance of this finding is seen in a reduced incidence of postoperative infection and collapse in the good lung. In lower lobectomy, we are still troubled by postoperative upper-lobe collapse. We do not think. that this is entirely due to soiling of the lung, for we have seen it in cases where no soiling of the lung has occurred and where oedema of the cut lower-lobe bronchus has encroached on the airway to the upper lobe.

The hanging position of the arm has given no trouble.

We have adopted the prone position with the flexed neck for all thoracotomies in which the lungs are not dry ; and, on account of the simplicity of the anaesthetic technique and the resultant stability of the mediastinum, we are increasingly using this position in other cases.

\section{REFERENCES}

Björkman, S. (1934). Acta med. scand., Supp. 56, $\overline{\text { p. } 118 .}$ Blair, L. G. (1941). Proc. R. Soc. Med., 35, 97.

Nosworthy, M. D. (1941). Proc. R. Soc. Med., 34, 479.

Overholt, R. H., et al. (1946). J. thorac. Surg., 15, 384. Penman, A. C. (1941). Tubercle, 22, 166. 